\title{
Characteristics of Spatial Connection Based on Intercity Passenger Traffic Flow in Harbin-Changchun Urban Agglomeration, China
}

Rong Guo, School of Architecture, Harbin Institute of Technology, Key Laboratory of Cold Region Urban and Rural Human Settlement Environment Science and Technology, Ministry of Industry and Information Technology, Harbin 150006, China

Tong Wu, School of Architecture, Harbin Institute of Technology, Key Laboratory of Cold Region Urban and Rural Human Settlement Environment Science and Technology, Ministry of Industry and Information Technology, Harbin 150006, China

Xiaochen $\mathrm{Wu}$, School of Architecture, Harbin Institute of Technology, Key Laboratory of Cold Region Urban and Rural Human Settlement Environment Science and Technology, Ministry of Industry and Information Technology, Harbin 150006, China

\begin{abstract}
With the continuous improvement of transportation facilities and information networks, the obstruction of distance in geographic space has gradually weakened, and the hotspots of urban geography research have gradually changed from the previous city hierarchy to the characteristics of urban connections and networks. As the main carrier and manifestation of elements, mobility such as people and material, traffic flow is of great significance for understanding the characteristics of spatial connection. In this paper, Harbin-Changchun agglomeration proposed by China's New Urbanization Plan (2014-2020) is taken as a research object. With the data of intercity passenger traffic flow including highway and railway passenger trips between 73 county-level spatial units in the research area, a traffic flow model is constructed to measure the intensity of spatial connection. Using social network analysis method, the characteristics of spatial connection of Harbin-Changchun urban agglomeration are analyzed, and the results are visualized with ArcGIS technique. The results show that the spatial connection of the Harbin-Changchun urban agglomeration based on traffic flow presents a distance attenuation effect that weakens in the directions of "Harbin-Daqing-Qiqihar-Mudanjiang" and "Changchun-Jilin-Tumen", and the connection strength of Harbin or Changchun districts and other spatial units is the strongest, follow by Daqing, Qiqihar or Jilin districts and other spatial units; the network is highly centralized, the core city has the dominant role on the whole network, and the connection between network nodes is not balanced; the connection nodes present a dualcore-edge hierarchical distribution; the network based on railway shift of Harbin-Changchun urban agglomeration can be divided into four subgroups,showing block distribution characteristic in Harbin-Changchun urban agglomeration. The research can provide support for the proposal of regionally coordinated sustainable development strategies of Harbin-Changchun urban agglomeration.
\end{abstract}

\section{Keywords}

Traffic Flow, Spatial Connection, Harbin-Changchun Urban Agglomeration

\section{Introduction}

Since the late 20th century, as globalization and informatization has accelerated and grown, the city constantly interacts with the outside world in the aspects of economy, society and culture[1]. In this 
context, the concept of "space of flows" has gradually replaced "space of place" as the basic theory for reviewing regional space interactions[2]. Research on regional spatial structures has also transformed from the central place model of rating scale to the city network model of factor relations[3-5]. With the rapid development of transportation infrastructure, the compression effect of "space-time distance" directly leads to the change of urban relative location and network role, and further shapes the regional development process from the perspective of flow space[6]. Aviation, railway, highway and other traffic flows have also become an important feature of regional spatial interaction from the perspective of flow space, and have been widely used in the study of urban network in different spatial scales such as global, national, provincial and urban agglomeration[7-9]. Traffic flow, as the main carrier and manifestation of population flow, logistics and other elements, can reflect the social and economic links between cities. which is of great significance for the study of the correlation and interaction between cities[10][11].

Research on the spatial characteristics of city networks has included three aspects. First, based on attribute data such as economy, population size and distance, most research methods have adopted modified gravity model to calculate economic link intensity and simulate interactions of social economic systems. Second, some research has applicated measured factor flow data such as population migration data and cargo flow data to reflect the city connection. Third, some scholars have used the parameter substitution method to replace the real connection between cities with information such as enterprise organization and passenger transport data[12][13]. However, based on the spatial attributes of places with spatial proximity characteristics, the "gravity model" takes some characteristic "stock" data of sample itself to describe the relationship between cities and towns and construct network relationship, which ignores the dynamic of the factor flow between cities. In China, due to the scarcity and difficulty of relational data acquisition, most of measured factor flow data are still insufficient in the aspects of sample size, continuity, timeliness and traceability. The research objects of applying measured factor flow data to study urban spatial relationship are mostly developed urban agglomerations, while the application of measured factor flow data in developing urban agglomerations such as Harbin-Changchun urban agglomeration and Guanzhong urban agglomeration is limited ${ }^{[14]}$. In China, considering difficulty of access to open data and limitation of static attribute data, some scholars began to use passenger shift data to reveal the characteristics and correlation of urban structure under the influence of passenger traffic flow (railway, highway, high-speed rail, aviation)[15].

As the microcosmic subject of urban agglomeration spatial connection, people's traffic commuting behavior is the external expression of production and life connection between cities. Therefore, traffic flow is more comprehensive and representative than other spatial flows. The urban spatial connection based on traffic flow reflects the network structure of urban agglomeration more accurately and objectively[16]. In terms of research scale, the existing urban agglomeration network research mostly stays at the city scale, and the accuracy can be guaranteed in the national urban network. However, for the urban agglomeration with small scope and few cities, the study of urban agglomeration network structure should be carried out at the district and county scale. In terms of research methods, social network analysis (SNA) provides a visual analysis tool for the study of urban agglomeration network structure. The core of SNA is to study the structure from the perspective of relationship. It describes the interaction between individuals in the network by quantitative indicators, which can not only reflect the position of individuals in the network structure, but also reveal the structural characteristics of the whole network.

The northeast of China, where Harbin-Changchun agglomeration is located, is one of the earliest areas in China's urbanization and industrialization, and the cradle of the birth of new China's heavy industry. However, since the 1980s, China's economic system has undergone important changes. Due to the strong inertia of planned economy, the old system can not adapt to the new development environment. At the same time, the northeast region is under the economic development law of "resource curse". The overall development began to decline. In 2014, The National New Urbanization Plan (2014-2020) emphasized the important position of Harbin-Changchun agglomeration. The plan considers that the development of Harbin-Changchun agglomeration has a stimulant effect for promoting the revitalization strategy of the old industrial base in Northeast China, the development of regional economic integration, and the 
transformation of resource-based cities[17]. It is urgent to carry out more sufficient academic research on Harbin-Changchun agglomeration. However, the existing studies mainly focus on the upgrading of industrial structure, the optimization of functional structure, and the measurement of economic ties. Thus, this paper takes Harbin-Changchun agglomeration as the research object. Based on the highway and railway passenger traffic flow data, and on the basis of multi-source traffic flow data fusion, this paper uses spatial analysis and social network analysis methods to measure the spatial connection pattern and spatial network characteristics of Harbin-Changchun agglomeration, in order to enrich the relevant research on the spatial characteristics of developing urban agglomerations, and provide useful reference for regional planning and regional policy-making.

\section{Study area and data sources}

Harbin-Changchun agglomeration (between $112^{\circ} 30^{\prime}-116^{\circ} 10^{\prime} \mathrm{E}$ and $29^{\circ} \quad 05^{\prime} \sim 31^{\circ} 50^{\prime} \mathrm{N}$ ) is located in the northeast of China, with an area of $320,534 \mathrm{~km} 2$. As shown in Figure 1, the Harbin-Changchun agglomeration covers 10 prefecture-level cities (Harbin, Daqing, Qiqihar, Suihua, and Mudanjiang in Heilongjiang province, and Changchun, Jilin, Siping, Liaoyuan, and Songyuan in Jilin Province), one autonomous prefecture (Yanbian Korean Autonomous Prefecture in Jilin Province) and 62 counties under the jurisdiction of the 10 prefecture-level cities. Harbin-Changchun agglomeration is located in the northern end of the vertical axis of Beijing-Harbin and Beijing-Guangzhou corridors in the national "two horizontal and three vertical" urbanization strategic pattern. It is the gateway of China's Northeast Asian export-oriented economic construction and has an important strategic position. The integrated development of Harbin great wall city group is the key to the revitalization of northeast old industrial base. Heilongjiang Province and Jilin Province have relatively mature planning for their urban system layout. The spatial strategy of urban development in Heilongjiang Province is "one circle driven, one belt first, three $\mathrm{Y}$ expansion". Jilin province put forward the spatial structure layout of "two districts, four axes and one belt" with the integration of Changchun-Jilin metropolitan area and Harbin-Daqing axis as the key points.These plans have great significance to promote the development of different regions in Harbin-Changchun agglomeration.

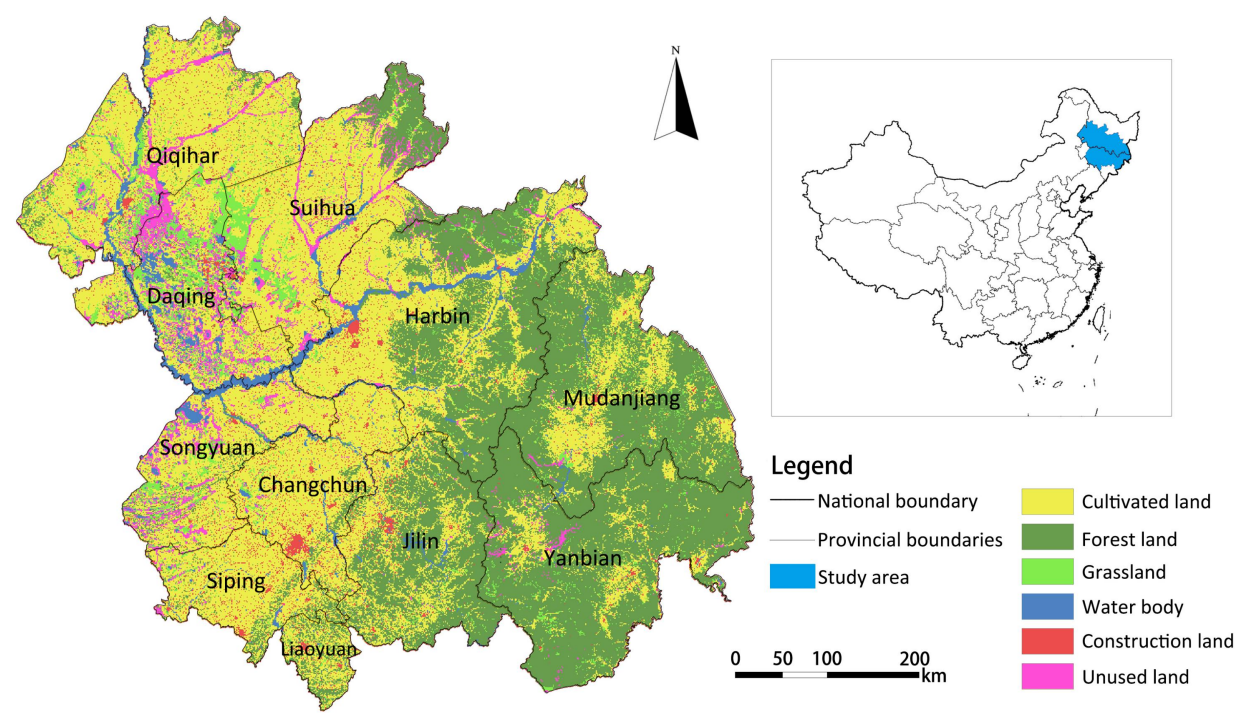

Figure 1. Land cover and geographical location of Harbin-Changchun urban agglomeration. Source: Painted by the author.

The original data of this research is the data of passenger transport frequency of vehicles and trains among 73 spatial research units of Harbin-Changchun urban agglomeration, including city districts, counties and county-level cities, so as to represent the spatial connection of transportation network from the perspective of highway and railway flow respectively. Among them, the data of highway bus frequency is from the 
automobile service website (https://www.checi.cn/) and China Road ticketing network (https://www.chuxing365.com/). The information of railway passenger transport, including the frequency data of ordinary trains and high-speed trains $(\mathrm{CRH})$, is all from the website of China Railway Customer Service Center (http:// www.12306.cn/ ). Due to the relatively fixed travel frequency of highway and railway, one day data is selected as the representative, and the data collection time is October 8, 2020. In order to ensure the scientificity and integrity of the data, random sampling, cross checking and other methods are used to correct and manually verify the data.

The train number information of 73 spatial research units is firstly inquired one by one to get the number of trains per day of each spatial research unit, and then all spatial research units are classified and summarized according to the train number to obtain the spatial unit data list of each train. In order to describe the inter city network association intuitively and scientifically, data transformation is needed, the directed network should be transformed into the undirected network. Therefore, based on the original shift data, the original (directed) relation matrix is transformed into (undirected) symmetric matrix for each type of traffic flow data, and the attribute values representing the relationship between cities are also transformed. Multi level feature is an important attribute of urban network spatial association. According to clustering algorithm (specifically natural fracture method), urban network spatial association under different traffic flow perspectives is drawn in turn. Relevant data preprocessing is operated in R language environment. The expression of urban network spatial association characteristics is realized in ArcGIS 10.2 software.

\section{Methodologies}

As illustrated in figure 2, we introduced a methodological scheme that summarizes the steps of the procedure used, which consists of key steps: (1) introducing the basic characteristics of Harbin-Changchun urban agglomeration, China, processing the basic traffic flow data, thereby constructing the OD flow matrix; (2) utilizing the Jenks natural breaks method and ArcGIS technique to analyze and visualize the spatial connection patterns including spatial connection hierarchy and distribution; (3) using social network analysis to detect spatial network characteristics through centrality, condensing subgroup and core-periphery analysis[18].

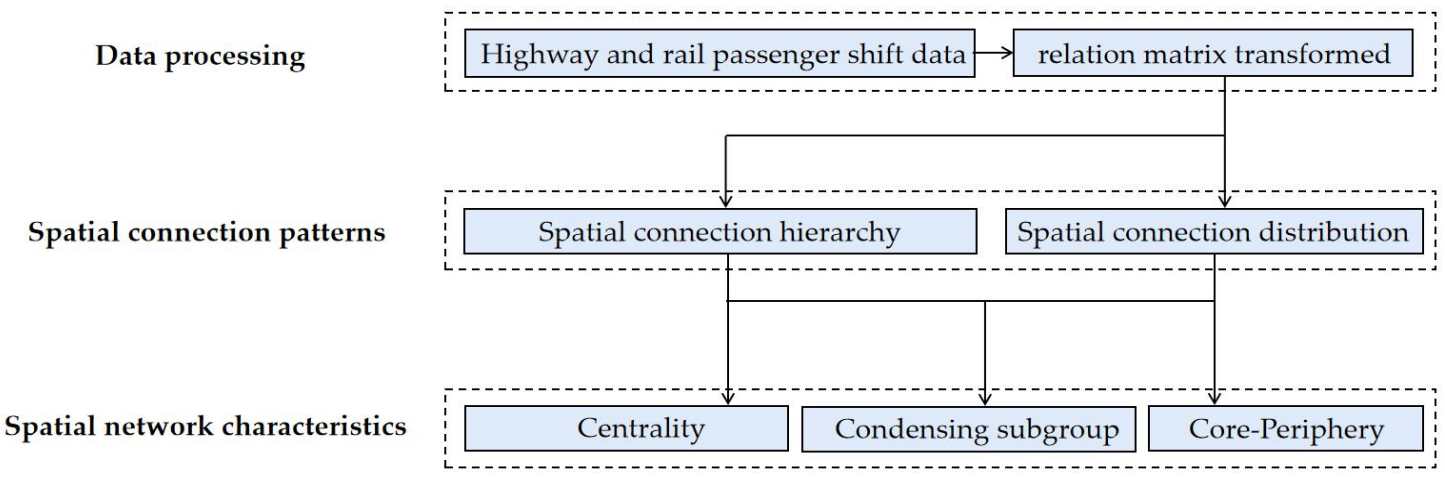

Figure 2. The methodology framework of the study. Source: Painted by the author.

\subsection{Spatial analysis method}

Based on the regional traffic flow data, the O-D (origin destination) matrix is constructed by using ArcGIS 10.2 software platform and network analyst module, and then the O-D network connection path is visualized. Referring to the relevant research, this paper uses the following indicators to measure the intensity of urban spatial connection.

Spatial connection strength $\left(M_{i j}\right)$ : connection strength refers to the degree of connection between cities and towns, which is obtained according to the weight of passenger frequency data of 
different transportation modes. Considering the difference of carrying capacity of different modes of transportation: 50 people are selected as the standard for bus; 1 soft sleeper, 8 hard sleeper and 8 hard seat cars are selected as the standard for general train, and the standard of 1200 people is defined; As for motor car and high-speed railway, 4 first-class cars, 5 second-class cars and 600 passenger loads are used as the standard. The connection strength of intercity passenger flow is measured and divided into 5 grades according to the connection strength based on natural breakpoint method. The calculation formula of traffic flow connection strength of urban agglomeration is as follows:

$$
\begin{gathered}
M_{i j}=50 A_{i j}+1200 B_{i j}+600 C_{i j}+600 D_{i j} \\
R_{i j}=M_{i j}+M_{j i}
\end{gathered}
$$

Where: $M_{i j}$ is the number of passenger flow from i research unit to j research unit; $R_{i j}$ is the traffic flow intensity between i research unit and j research unit; $A_{i j}, B_{i j}, C_{i j}$ and $D_{i j}$ are the number of intercity bus, ordinary train, EMU and high-speed rail from i research unit to $\mathrm{j}$ research unit.

\subsection{Social network analysis method}

Based on the spatial connection network of urban agglomerations, with the help of UCINET 6.0 software platform, this paper measures the spatial network characteristics of Harbin-Changchun urban agglomeration from three aspects: network centrality, core edge structure and agglomerative subgroups. These analyses cover different angles such as point, line, surface and group, which can comprehensively reflect the structural characteristics of flow space network[19][21].

If a city is directly related to many other cities, then the city is in a central position and has greater power. Therefore, it can be directly measured by the number of related points. The higher the centrality of point degree is, the more central the city is in the network[20]。The calculation formula is as followed:

$$
C_{D}\left(C_{j}\right)=d\left(C_{j}\right) /(n-1)
$$

Where $C_{D}$ is the centrality of node ${ }^{j}$ and $d\left(C_{j}\right)$ is the absolute centrality (actual degree) of node ${ }^{j}$.

Core periphery structure: core edge structure reveals the position and role of each city node in the urban agglomeration network, which has become the research paradigm of network structure analysis. In this study, the continuous core edge model is used to determine the ownership of the core area and the edge area of urban nodes in the network based on the coreness[21]。

Agglomerative subgroup is a concept of subgroups with broad meanings. It generally refers to the subsets of members with strong, direct, close, frequent or positive relationships among members. In this paper, the agglomerative subgroup analysis is based on the traffic flow connection strength between cities, and discusses the small group agglomeration phenomenon, so as to reflect the intimate relationship between cities, and then judge the organizational structure of urban connection network in urban agglomeration.

\section{Results}

\subsection{Spatial connection patterns of Harbin-Changchun urban agglomeration}

According to the calculation method of traffic flow connection strength mentioned above, the OD data of traffic flow among 73 research units in Harbin Changcheng city group is calculated. A total of 2628 passenger traffic flow links are obtained. The applied railway passenger transport shift data are shown in the table, and the spatial distribution pattern of the obtained links is shown in the figure.

Table 1. Railway passenger shift data

Total High-speed Motor Express Fast train ordinary




\begin{tabular}{lllllll}
\hline & & train & train & train & & train \\
\hline Theoretical quantity & 5256 & 5256 & 5256 & 5256 & 5256 & 5256 \\
\hline Actual quantity & 684 & 132 & 146 & 40 & 184 & 112 \\
\hline Realization rate (\%) & 13.01 & 2.51 & 2.78 & 0.76 & 3.50 & 2.13 \\
\hline $\begin{array}{l}\text { Train working times/spatial } \\
\text { connction }\end{array}$ & 7.20 & 8.11 & 2.07 & 1.60 & 4.45 & 1.61 \\
\hline
\end{tabular}

Note: all train numbers include not only general express, express, express, EMU and HSR in the table, but also intercity trains, tourist trains and through trains.

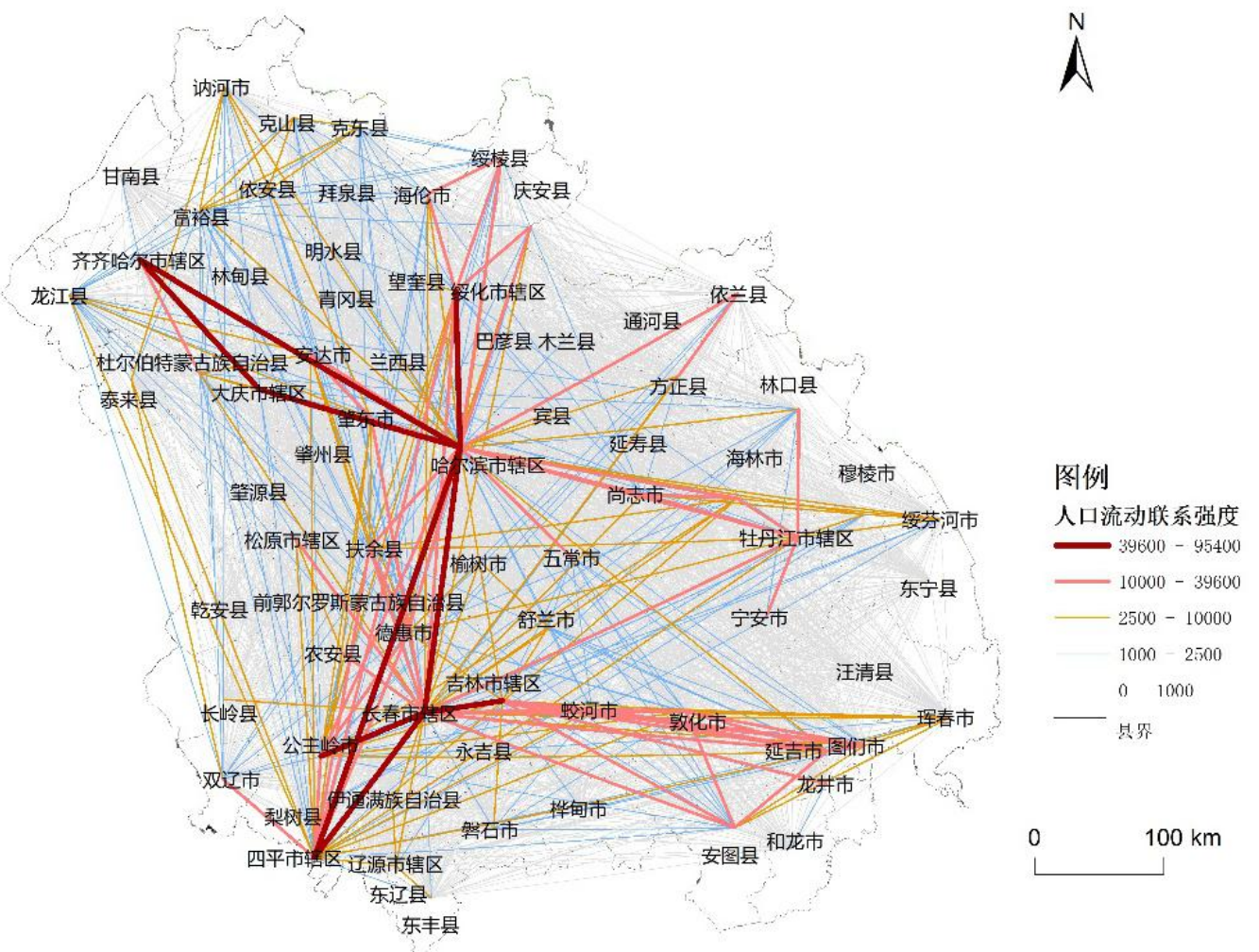

Figure 3. Spatial distribution pattern of Harbin-Changchun urban agglomeration. Source: Painted by the author.

The figure shows the connection strength and spatial distribution pattern of traffic flow among 73 spatial research units of Harbin-Changchun urban agglomeration based on the formula mentioned above. On the whole, the passenger flow connection among the 73 research units in the Harbin-Changchun urban agglomeration shows the characteristics of "more in the north-south direction and less in the east-west direction", and the traffic flow model presents a "double center radiation" and T-shaped pattern. Taking the districts of Harbin and Changchun as the centers, The study units are connected radially, forming a spatial connection axis with obvious grade difference.

From a local point of view, based on the sub pattern of the flow of traffic in Harbin-Changchun urban agglomeration, the district can be divided into two sub regions: the northern district takes Harbin as the center, showing a "fan-shaped" spatial connection structure; Daqing, Qiqihar, Suihua and Mudanjiang form a "fan" together with Harbin; the southern district takes Changchunas the center, showing a "radial" spatial pattern. The "radial" spatial connection structure extends to Gongzhuling and Siping in the southwest, takes Jilin as the bridge in the east, and stretches along Jiaohe, Dunhua and Yanji connection axis in the southeast direction, forming a close contact corridor of traffic flow. The spatial connection of the Harbin-Changchun urban agglomeration based on traffic flow presents a distance attenuation effect that weakens in the directions of "Harbin-Daqing-Qiqihar-Mudanjiang" and "Changchun-Jilin-Tumen"; the 
direction of the connection clearly points to the Heilongjiang' s provincial capital Harbin and Jilin' $s$ provincial capital Changchun, which proves that the agglomeration effect with Harbin and Changchun districts as the core is significant; the connection strength of Harbin or Changchun districts and other spatial units is the strongest, follow by Daqing, Qiqihar or Jilin districts and other spatial units. However, counties and cities in the border areas of Heilongjiang and Jilin provinces have the lowest connection strength with other counties and cities, indicating that there are obvious regional differences in the spatial connection level of the Harbin-Changchun urban agglomeration.

According to the grade distribution of spatial connection axes, there are 9 primary connection axes, among which Changchun-Siping district has the strongest spatial connection, and the connection strength value reaches 95400. Even though the connection strength between Harbin and Changchun is relatively weaker. Changchun-Siping connection strength reaches the first level, mainly because the spatial connection depends on railway passenger transport. It is worth noting that "Harbin-Daqing-Qiqihat" connection and "Harbin-Changchun-Siping" connection form two triangle closed loops at the first level. There are 124 secondary connection axes, which extend outward along the primary axis. The spatial distribution of the connecting axis of the secondary passenger flow is basically consistent with the trend of the regional highspeed road network and railway trunk lines, which reflects the supporting role of transportation infrastructure for the spatial connection of Harbin-Changchun urban agglomeration. It should be noted that the traffic flow connection intensity generally shows the spatial proximity effect, that is, the two research units closer in space are more closely connected. In addition, there are differences in traffic flow connection capacity among different administrative level research units, and the link between "county to county" is weaker than that of "county to city". This phenomenon can not be explained by spatial proximity effect, because administrative boundary barrier weakens the attenuation effect of connection strength in spatial distance from the system and relationship level.

\subsection{Spatial network characteristics of Harbin-Changchun urban agglomeration}

This paper analyzed the point centrality of each city in Harbin-Changchun urban agglomeration, so as to determine the "Centrality" capacity of each city within the scope of urban agglomeration. The absolute centrality and relative centrality of each city were analyzed by UCINET. The descriptive statistics results of centrality were as follows:

Table 2 The descriptive statistics results of centrality

\begin{tabular}{|c|c|c|c|}
\hline \multicolumn{4}{|c|}{ DESCRIPTIVE STATISTICS } \\
\hline & Degree & NrmDegree & Share \\
\hline 1 Mean & 128682 & 0.956 & 0.014 \\
\hline 2 Std Dev & 223336 & 1.659 & 0.023 \\
\hline 3 Sum & 9522500 & 70.740 & 1.000 \\
\hline $4 \quad$ Variance & 49879248896 & 2.753 & 0.001 \\
\hline $\begin{array}{ll}5 & \text { SSQ }\end{array}$ & 4916442562560 & 271.321 & 0.054 \\
\hline $\begin{array}{ll}6 & \text { MCSSQ }\end{array}$ & 3691064328192 & 203.697 & 0.041 \\
\hline $7 \quad$ Euc Norm & 2217305 & 16.472 & 0.233 \\
\hline 8 Minimum & 0 & 0 & 0 \\
\hline 9 Maximum & 1244650 & 9.246 & 0.131 \\
\hline
\end{tabular}

Network Centralization $=8.52 \%$

Heterogeneity $=5.42 \%$. Normalized $=4.13 \%$ 


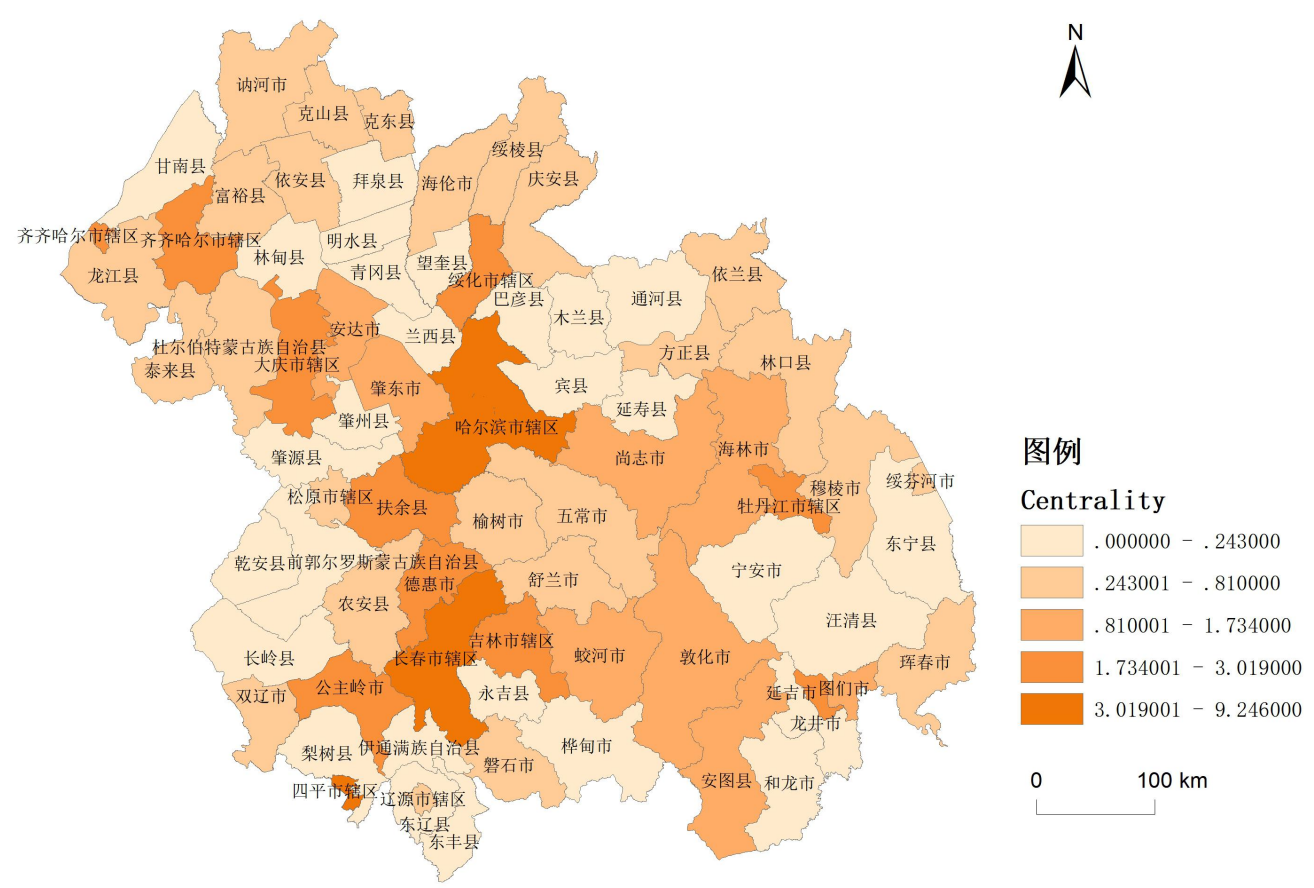

Figure 4. The centrality of Harbin-Changchun urban agglomeration. Source: Painted by the author.

The calculated urban centrality was visualized by ArcGIS natural fracture method, as shown in the figure 4 . The average centrality of traffic flow network is 0.956 , which indicates that the network node connection is at a low level. Harbin, Changchun, Gongzhuling, Daqing and Qiqihar are the top five cities in the order of centrality. The centrality of Harbin reaches 9.246, which means that Harbin has direct contact with other cities in Harbin-Changchun urban agglomeration, which highlights the core position of Harbin in HarbinChangchun urban agglomeration. It can be seen from the figure that the spatial distribution of centrality shows the characteristics of "high in the middle and low in the edge" on the whole, showing the strike characteristics of the axial belts of Harbin-Daqing-Qiqihar, Harbin-Changchun and Changchun-Jilin-Yanji. The cities with higher centrality than the average are concentrated in the central area of Harbin-Changchun urban agglomeration, such as Shangzhi, Mudanjiang, Jiaohe, Dunhua, Hailin, etc., which indicates that the influence of the central region on the network structure of the urban agglomeration is stronger than that of other cities. On the whole, spatial network of Harbin-Changchun urban agglomeration has formed a structure with Harbin and Changchun as the absolute core. The network is highly centralized, the core city has the dominant role on the whole network, and the connection between network nodes is not balanced. 


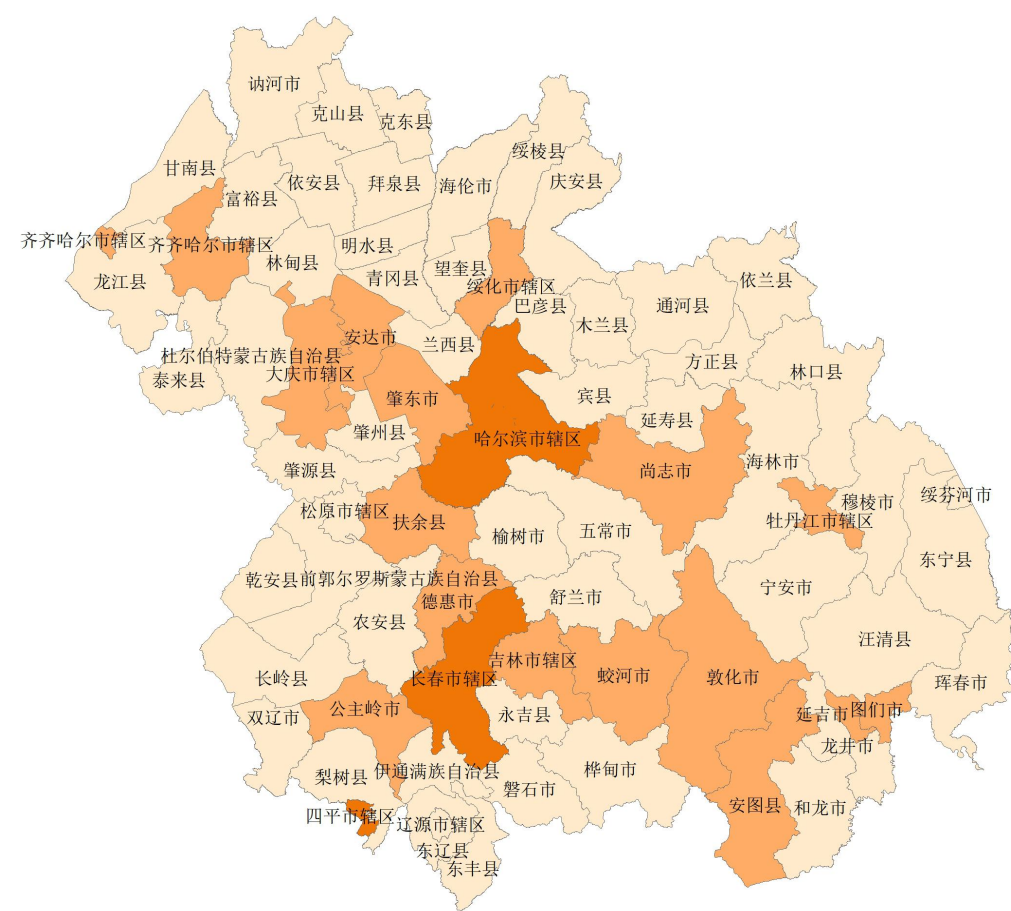

$\AA$

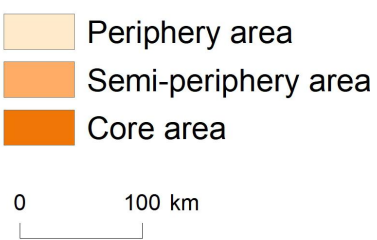

Figure 5. The core-periphery structure of Harbin-Changchun urban agglomeration. Source: Painted by the author.

Core-periphery structure analysis: in this paper, the core-periphery model of UCINET software is used to analyze the spatial network characteristics of Harbin-Changchun urban agglomeration, and the core area, semi-periphery area and periphery area are identified based on this model. In the spatial network based on traffic flow connection (Fig. 5), the fitting value of the result is 0.81 , which has high reference value. There are three core cities, Harbin, Changchun and Siping, which are in line with the top three cities in traffic network centrality ranking. Yushu and Shulan, as cities connecting the west and east of Harbin-Changchun urban agglomeration, have no significant bridge effect and radiation effect. There are 15 semi-periphery cities, which are distributed along the axis of northwest-southeast and northeast-southwest, with significant spatial adjacency effect. Edge cities are distributed in the periphery of the agglomeration. Some cities are adjacent to the core areas, such as Lanxi, Wuchang and Zhaozhou. Affected by the "shadow effect of the core cities, these cities are in the marginal areas of the connection network, and the external traffic links depend on the turnover of the core cities. On the whole, the spatial network of Harbin-Changchun urban agglomeration embodies the structural characteristics of "core-periphery", that is, strong connection between core cities, weak connection between peripheral cities, and general connection between core cities and peripheral cities. 


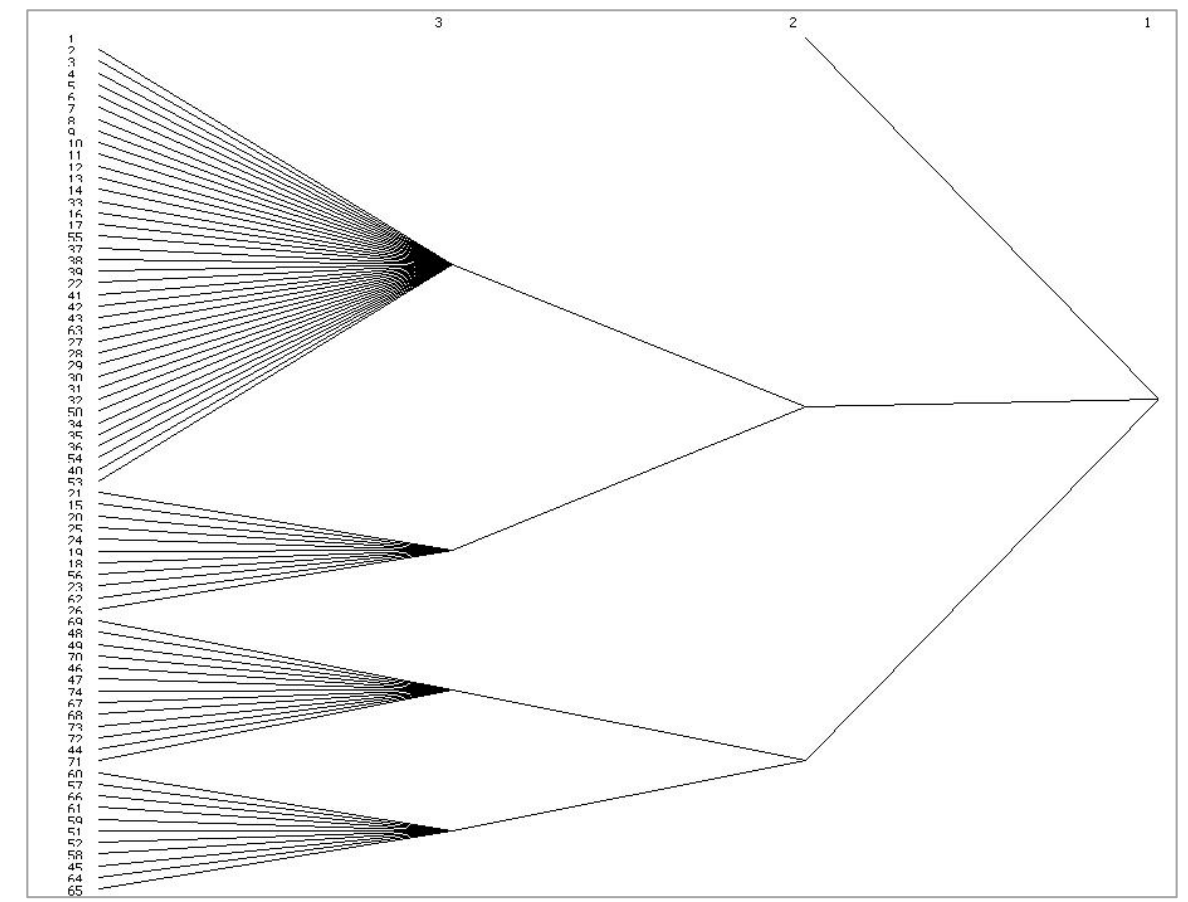

Figure 6. Agglomerative subgroup of Harbin-Changchun urban agglomeration. Source: Painted by the author.

Based on the iterative correlation convergence method (Concor) algorithm in ucinet6.0 software, the internal of traffic flow connection network of Harbin-Changchun urban agglomeration is studied from the micro perspective, and the agglomerated subgroup structure diagram of traffic flow connection network is obtained as shown in the figure 6. For convenient observation, the agglomerated subgroups at the third level are drawn by the visualization function of ArcGIS, as shown in figure 7.

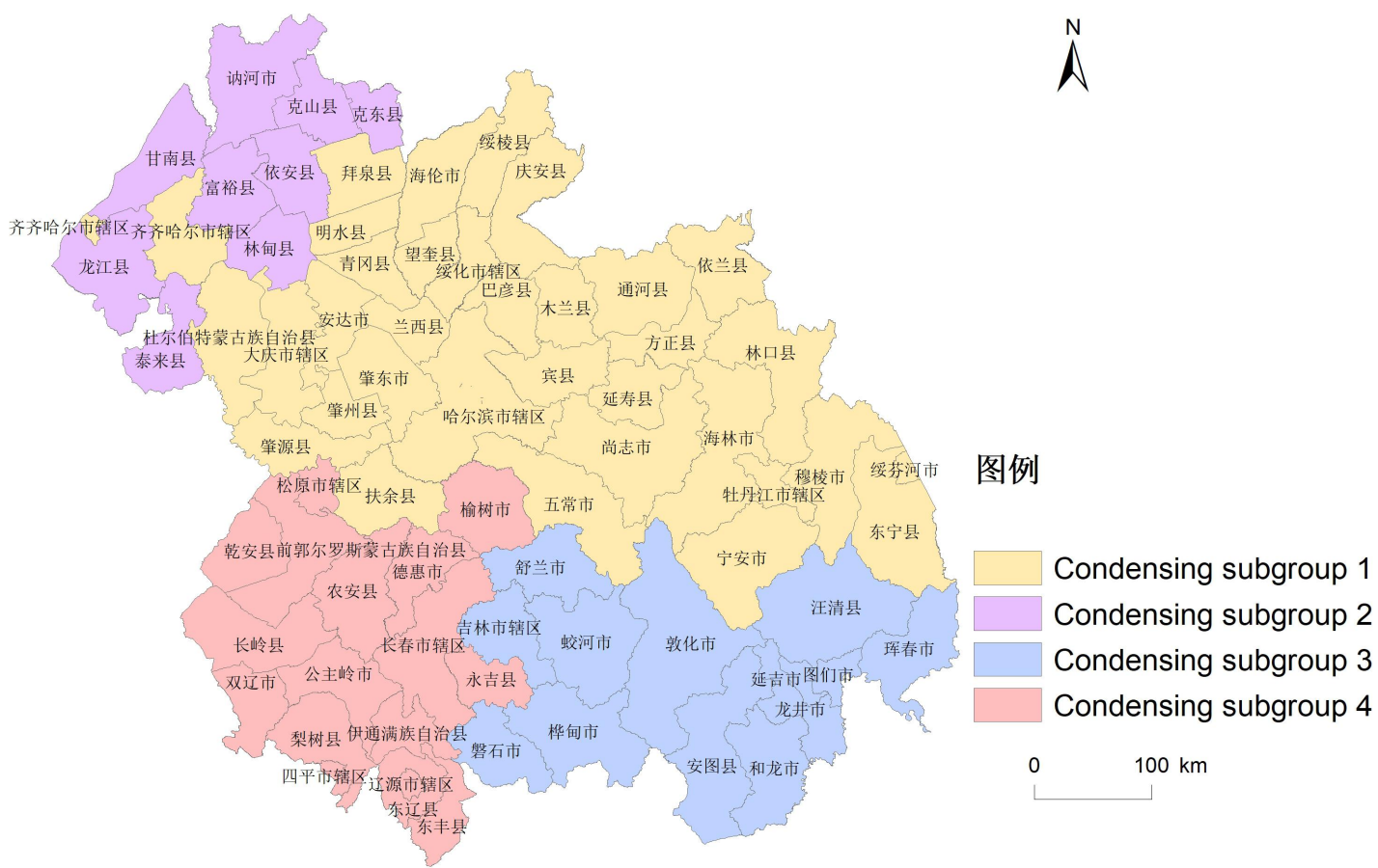

Figure 7. Spatial distribution of agglomerative subgroups. Source: Painted by the author.

By observing the results of agglomerating subgroups in the graph, the network based on traffic flow of Harbin-Changchun urban agglomeration can be divided into four subgroups: the first is the agglomerative 
subgroup composed of Harbin, Mudanjiang, Daqing, Suihua and Qiqihar; the second is the agglomerative subgroup formed by most counties of Qiqihar; the third is composed of most counties of Jilin; the fourth is the agglomerative subgroup of Changchun, Siping, Liaoyuan and Songyuan. In the first agglomerative subgroup, the traffic flow of Harbin and the counties adjacent to Songyuan and Changchun are more frequent, which indicates that the railway traffic flow between prefecture level cities is relatively close. In the second, third and fourth agglomerations, counties tend to be closer in the same city and to themselves. The formation of agglomerative subgroups shows that the highway traffic flow among counties in the city is closely related. From the overall perspective of agglomerative subgroups, the formation and division of agglomerative subgroups are affected by geographical location and other factors, some of which are sheet distribution, some are not adjacent or cross the administrative boundaries, showing block distribution characteristic in Harbin-Changchun urban agglomeration.

\section{Discussion}

The analysis of spatial connection and network characteristics is of great significance for further optimizing the inter city network structure of Harbin-Changchun urban agglomeration and promoting the high-quality development of Harbin-Changchun urban agglomeration. Therefore, this paper has two enlightenments: (1) Regional planning should focus on strengthening the leading role of the core nodes, and consolidate the leading position of Harbin and Changchun. The competition and cooperation between the central cities should be guided. The government should give full play to the "agglomeration effect" to drive the development of the surrounding small and medium-sized cities, and also should strive to avoid the "shadow of Agglomeration" between the central cities. In addition, small and medium-sized cities should take the initiative to connect with the development of central cities, such as strengthening the comprehensive competitiveness of Mudanjiang, Suihua and Yanji, so as to integrate these cities into the development process of urban agglomerations. (2) We should speed up the construction of high-speed traffic network and guide the spatial distribution and lead traffic flow reasonably. The construction of intercity railway should speed up, especially in the areas with strong spatial correlation of traffic flow. The construction of high-speed traffic and the guiding role of traffic need to be taken seriously. We should focus on building regional central cities, get through the connection channels between central cities and small and medium-sized cities, ensure that the inter city connection channels of central cities expand to small and medium-sized cities in urban agglomeration. The benefit range and scope brought by the growing economic effect and node agglomeration effect of regional central cities need to be expanded. The government should promote the integration of peripheral cities of urban agglomerations into the development network of urban agglomerations to form a closer inter city spatial network.

This study takes Harbin-Changchun urban agglomeration as the research area. Although it has the general characteristics of urban agglomeration, due to its own characteristics, whether the traffic flow law of Harbin-Changchun urban agglomeration can be applied to other urban agglomerations needs further discussion. In addition, there are great differences in the development degree of differenet urban agglomerations in China, and the development level of urban agglomerations is also different.

\section{Conclusions}

Urban agglomerations have the largest growth potential and have become the most dynamic areas in Chinese economic development. Along with the transformation of the basic regional competition units, the administrative fortresses between cities have been broken. Economic and human activities have become increasingly frequent, making factor flow become the main form of connecting the administrative units of urban agglomerations. In order to enrich the relevant research on the spatial characteristics of developing urban agglomerations, and provide useful reference for regional planning and regional policy-making, this paper analyzes the spatial connection pattern and spatial network structure characteristics of HarbinChangchun urban agglomeratio with the help of the ArcGIS10.2 and UCINET6.0 platform. The main conclusions are as follows: 
The spatial connection of the Harbin-Changchun urban agglomeration based on traffic flow presents a distance attenuation effect that weakens in the directions of "Harbin-Daqing-Qiqihar-Mudanjiang" and "Changchun-Jilin-Tumen"; the direction of the connection clearly points to the Heilongjiang' s provincial capital Harbin and Jilin' s provincial capital Changchun, which proves that the agglomeration effect with Harbin and Changchun districts as the core is significant; the connection strength of Harbin or Changchun districts and other spatial units is the strongest, follow by Daqing, Qiqihar or Jilin districts and other spatial units. However, counties and cities in the border areas of Heilongjiang and Jilin provinces have the lowest connection strength with other counties and cities, indicating that there are obvious regional differences in the spatial connection level of the Harbin-Changchun urban agglomeration.

The centrality analysis result shows that the spatial network of Harbin-Changchun urban agglomeration has formed a structure with Harbin and Changchun as the absolute core. The network is highly centralized, the core city has the dominant role on the whole network, and the connection between network nodes is not balanced. The core-periphery analysis result shows that the spatial network of Harbin-Changchun urban agglomeration embodies the structural characteristics of "core-periphery", that is, strong connection between core cities, weak connection between peripheral cities, and general connection between core cities and peripheral cities. The results of agglomerating subgroups show that the network based on traffic flow of Harbin-Changchun urban agglomeration can be divided into four subgroups, the formation and division of agglomerative subgroups are affected by geographical location and other factors, some of which are sheet distribution, some are not adjacent or cross the administrative boundaries, showing block distribution characteristic in Harbin-Changchun urban agglomeration.

\section{References}

[1]Du S., Zhang Z. (2020) 'Research on the relationship of Yangtze River Delta urban network based on railway passenger traffic flow', Urban journal, 041(001), p90-97.

[2]Ke W., Chen W., Yu Z. (2017) 'Uncovering Spatial Structures of Regional City Networks from Expressway Traffic Flow Data: A Case Study from Jiangsu Province, China’, Sustainability, 9(9).

[3]Castells, M. (2010) 'Globalisation, Networking, Urbanisation: Reflections on the Spatial Dynamics of the Information Age', Urban Studies, 47(13), p2737-2745.

[4]Castells, M. (1996) The Rise of the Network Society. UK: Blackwell.

[5]Batty, M. (1989) 'Cities as Information Networks', J.env.cons.eng, 19(8), p761-775.

[6]Yang, Z., Algesheimer, R. (2016) 'Tessone, C.J. A comparative analysis of community detection algorithms on artificial networks', Sci. Rep, 6, p30750.

[7]Feng X.H., Xiu C.L., Bai L.M. (2019) 'Urban centrality and its influencing factors in Jilin Province from the perspective of highway traffic flow', Economic geography, 39(01), p64-72.

[8]Smith D.A., Timberlake M.F. (2001) 'World city networks and hierarchies, 1977-1997: an empirical analysis of global air travel links', American Behavioral Scientist, 44(10), p1656-1678.

[9]Guimera R., Mossa S., Turtschi A. (2005) 'The worldwide air transportation network: Anomalous centrality, community structure, and cities' global roles', Proceedings of the National Academy of Sciences, 102(22), p7794-7799.

[10]Du S., Zhang Z.H. (2020) 'Research on the relationship of Yangtze River Delta urban network based on railway passenger traffic flow', Urban journal, 041(001), p90-97.

[11]Jiao, J.J., Wang, J.E., Jin, F.J. (2016) 'Impact of high-speed rail on inter-city network based on the passenger train network in China, 2003-2013', Acta Geogr, 71, p265280.

[12] Taylor, P., Hoyler, M., Verbruggen, R. (2010) 'External urban relational process: Introducing central flow theory to complement central place theory’, Urban Stud, 47, p2803-2818. 
[13]Timberlake M. (2008) 'The Polycentric Metropolis: Learning from Mega-City Regions in Europe', Journal of the American Planning Association, 74(3), p384-385.

[14] Li, S.M., Shum, Y.M. (2001) 'Impacts of the national trunk highway system on accessibility in China', J. Transp. Geogr, 9, p39-48.

[15]Ke W., Chen W.,Yu Z.(2017) 'Uncovering Spatial Structures of Regional City Networks from Expressway Traffic Flow Data: A Case Study from Jiangsu Province, China', Sustainability, 9(9).

[16]Chen H., Quan D.J., Zhao X.Z. (2019) 'Study on spatial connection network of Guanzhong Urban Agglomeration Based on traffic flow’, Resource development and market, 035(002), p236-242.

[17]Guo R., Wu T., Liu M.R. (2019) 'The Construction and Optimization of Ecological Security Pattern in the Harbin-Changchun Urban Agglomeration, China', International Journal of Environmental Research \& Public Health, 16(7).

[18]Jiang L.L., Yuan J.D., Di Y.S., Liu Y.Q. (2020) 'Urban spatial structure and the definition of the hinterland of local central cities in Jilin Province', Geoscience, 40 (08), p1319-1327.

[19]Zhang W.S. (1992) Spatial transportation linkage: theoretical research, empirical analysis and prediction method. China: Railway Press.

[20]Feng X.H., Xiu C.L., Zhong Y.X. (2017) 'The network level of cities in the Yangtze River Economic Belt: an analysis based on the perspective of railway and highway passenger transport', Urban issues, 07, p20-28. 